\title{
Multiplexing of Fiber-Optic Long-Period Grating-Based Interferometric Sensors
}

\author{
Richard P. Murphy, Stephen W. James, and Ralph P. Tatam
}

\begin{abstract}
Two long-period gratings (LPGs) fabricated in series in an optical fiber form a Mach-Zehnder interferometer, producing a sinusoidal channelled spectrum within the characteristic LPG attenuation bands, which has the appearance of an interference fringe pattern. The phase of the fringes is sensitive to changes in the surrounding environmental parameters, such as refractive index. Fabrication of a number of identical cascaded LPG pairs with different separations produces independent fringe patterns of different frequencies within the attenuation bands. The application of basic Fourier techniques to analyze the transmission spectrum allows the phase of each fringe pattern to be determined independently, facilitating the differentiation of external effects acting on each interferometer.
\end{abstract}

Index Terms-Multiplexing, optical-fiber long-period gratings (LPGs), sensors.

\section{INTRODUCTION}

O PTICAL-FIBER long-period gratings (LPGs) are the subject of considerable research interest for use as sensor elements. LPGs exhibit sensitivity to environmental parameters such as strain, temperature, curvature, and refractive index of the surrounding material [1]. A limitation of LPG-based sensor systems is the difficulty associated with multiplexing serial sensor arrays. In a recent report, this problem was addressed by using the reflection from matched fiber Bragg gratings to illuminate an array of two LPGs of different periods. Derivative spectroscopy techniques were implemented to facilitate the interrogation and demultiplexing of the LPG sensors, which were deployed as bend sensors [2]. Here, the multiplexing of a serial array of LPG-based Mach-Zehnder interferometers (MZIs) by the application of simple Fourier transform techniques to the transmission spectrum is explored.

The formation of an intrinsic MZI by cascading two LPGs has been shown to produce a sinusoidal channelled spectrum within the attenuation bands that characterize the transmission spectrum of an LPG [3], which has the appearance of an interference fringe pattern. Cascaded LPGs have been used to sense bending [3], external index of refraction [4], [5], temperature, and transverse load [5]. The influence of nanostructured coatings on the phase of the fringes has been investigated for sensing applications [6].

Manuscript received April 20, 2006; revised December 1, 2006. This work was supported by the Engineering and Physical Science Research Council U.K.

The authors are with the Engineering Photonics Group, School of Engineering, Cranfield University, MK43 0AL Cranfield, U.K. (e-mail: r.p.murphy@ cranfield.ac.uk; s.w.james@cranfield.ac.uk; r.p.tatam@cranfield.ac.uk).

Color versions of one or more of the figures in this paper are available online at http://ieeexplore.ieee.org.

Digital Object Identifier 10.1109/JLT.2006.889670
The frequency of the fringes is determined predominantly by the separation of the LPGs, while the phase is influenced by measureand-induced changes in the effective index of the cladding modes. Thus, if an array of cascaded LPG sensors were fabricated, each with a different LPG separation, it would be possible to identify each sensor element from the frequency of the fringes in the transmitted optical spectrum, while the phase of the fringes would allow the measureand to be monitored.

The technique has analogies with the frequency-modulated continuous wave technique used to multiplex fiber-optic MZIs, in which the optical frequency of the source used to illuminate a serial array of MZIs with different path length imbalances is modulated [7], [8]. This produces a distinct carrier frequency for each MZI, which is dependent on the path length imbalance and the frequency excursion of the source. In this paper, the optical frequency scan is achieved by illuminating the LPG-based MZI array using a broadband optical source and monitoring the transmission spectrum using an optical spectrum analyzer. Analyzing the Fourier transform of the transmission spectrum allows the carrier frequencies associated with each MZI to be distinguished and the phase to be determined.

\section{PRinciple of the Technique}

\section{A. Cascaded LPGs}

An LPG is a periodic modulation of the propagation constants of the modes of an optical fiber, which is formed by a number of means, particularly UV-induced modulation of the refractive index of the core of the fiber. The period of the modulation is typically in the range of $100-1000 \mu \mathrm{m}$ and facilitates coupling of energy between the propagating mode of the core of the fiber and a discreet set of forward-propagating cladding modes. Coupling takes place at wavelengths determined by the phase matching condition, which is dependent on the product of the difference between the effective indexes of the core and cladding modes with the period of the modulation [1]. Thus

$$
\lambda_{i}=\left(n_{\mathrm{eff}}(\lambda)-n_{\mathrm{cl}}^{i}(\lambda)\right) \Lambda
$$

where $n_{\mathrm{eff}}(\lambda)$ is the effective refractive index of the propagating core mode at wavelength $\lambda, n_{\mathrm{cl}}^{i}(\lambda)$ is the refractive index of the $i$ th cladding mode, and $\Lambda$ is the period of the LPG. The high attenuation of the cladding modes results in the presence within the transmission spectrum of a set of attenuation bands at the central wavelengths defined by (1). Thus, a single LPG introduces a number of relatively broad spectral features into 


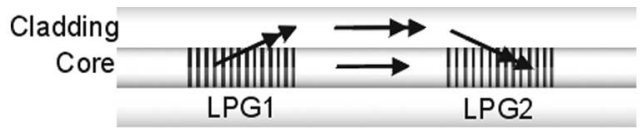

(a)

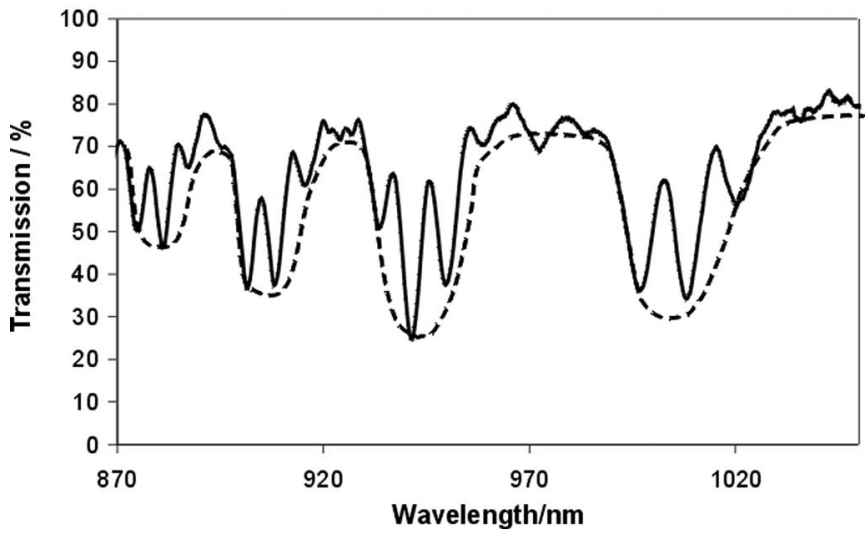

(b)

Fig. 1. (a) Operation of cascaded LPGs as an MZI. (b) Interference fringes in the attenuation bands.

the transmission spectrum of the optical fiber, which, when coupled with the large sensitivity of the central wavelength of the attenuation bands to environmental parameters, can limit the practicality of the use of wavelength-division multiplexing techniques for serial arrays of LPG sensors.

The fabrication of in-series or cascaded LPGs results in the formation of an MZI, producing interference fringes within the attenuation bands, as illustrated in Fig. 1. The first LPG acts to couple light to a cladding mode. Light then propagates to the second LPG via two routes, namely 1) in the core and 2) in the cladding. At the second LPG, the cladding mode is coupled back into the core. By virtue of the difference between the effective refractive indexes of the core and cladding modes, the light coupled into the core by the second LPG is phase shifted with respect to the light that propagated through the core, giving rise to the interference pattern.

The transmission spectrum of cascaded LPGs is described by [9]

$$
\begin{aligned}
I= & I_{\text {core }}+\alpha I_{\text {cladd }}^{i}-2 \sqrt{\alpha I_{\text {core }} I_{\text {cladd }}^{i}} \\
& \times \cos \left(\frac{2 \pi}{\lambda}\left(n_{\text {effcore }}-n_{\text {effladd }}^{i}\right) L+\Phi\right) \\
\Phi= & 2 \tan ^{-1}\left(\frac{\Delta \beta}{2 s} \tan s d\right)-\Delta \beta d \\
\Delta \beta= & \frac{2 \pi}{\lambda}\left(n_{\text {effcore }}-n_{\text {effcladd }}^{i}\right)-\frac{2 \pi}{\lambda}
\end{aligned}
$$

where $I_{\text {core }}$ and $I_{\text {cladd }}^{\mathrm{i}}$, and $n_{\text {effcore }}$ and $n_{\text {effcladd }}^{\mathrm{i}}$, are the intensities and propagation constants, respectively, of the core and cladding modes, $\alpha$ is the attenuation of the cladding mode, $d$ is the grating length, $L$ is the separation of the LPGs, and $s$ depends on coupling coefficient $\kappa$ and detuning $\Delta \beta, \mathrm{s}^{2}=$ $\kappa \kappa^{*}=(\Delta \beta / 2)^{2}$. The phase of the interference pattern is depen-

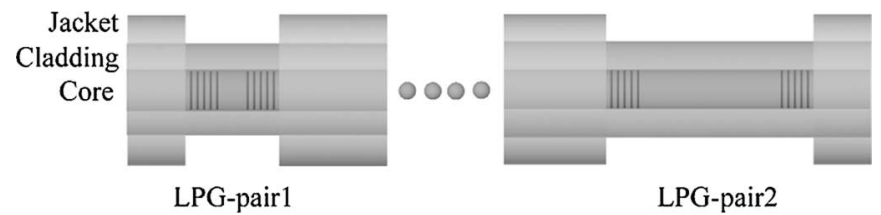

Fig. 2. Optical fiber containing four LPGs.

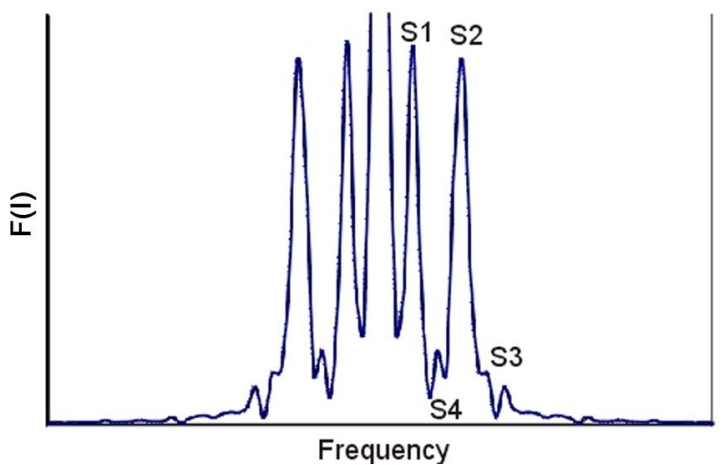

Fig. 3. Fourier transform of the intensity spectrum $F(I)$ of an optical fiber containing two cascaded LPG pairs of differing separations. Si, $i=1-4$ labels the significant frequency components present. The cascaded LPG pairs form two independent MZIs.

dent on a phase difference imposed by the LPG $\Phi$ and on the optical path difference between the core and cladding modes.

Fig. 2 illustrates the proposed multiplexed LPG MZI system. Two or more cascaded LPG pairs are fabricated in a single length of fiber, with each LPG pair having a different physical separation to produce fringes of different frequencies. The LPGs all have identical properties, such that the attenuation bands overlap. For an individual cascaded LPG pair, the length of fiber separating the LPGs has its polymer buffer jacket removed to allow the cladding modes to propagate between the LPGs. Between each LPG pair, the presence of the buffer jacket ensures that the cladding modes are stripped and that the interferometers are independent.

\section{B. Signal Processing}

Assuming that the LPGs are identical and have a 3-dB coupling strength and that the attenuation of the cladding modes can be neglected, the resulting intensity pattern can be described by

$$
\begin{aligned}
I= & I_{0}(\lambda)\left(1-\cos \left(\frac{2 \pi}{\lambda}\left(n_{\text {effcore }}-n_{\text {effcladd }}^{i}\right)_{1} L_{1}+\Phi\right)\right. \\
& \left.\times\left(1-\cos \left(\frac{2 \pi}{\lambda}\left(n_{\text {effcore }}-n_{\text {effcladd }}^{i}\right)_{2} L_{2}+\Phi\right)\right)\right)
\end{aligned}
$$

where $I_{0}(\lambda)$ represents the spectral form of the attenuation band envelope, $L_{1}$ and $L_{2}$ are the fiber lengths separating LPG pairs 1 and 2, respectively, and $\Phi$ is the phase imposed by the LPGs. The Fourier transform of such an experimentally obtained intensity pattern is illustrated in Fig. 3. Four distinct peaks are visible on each side of the central DC peak, which is truncated in amplitude in the interest of clarity. 
These four peaks represent the four frequencies present in the intensity pattern. Each MZI introduces a unique frequency to the spectrum, S1 and S3, respectively, determined by the physical separation of the component LPGs. The sum and difference frequency components S4 and S2, respectively, are also present. As for all frequency-based interferometer multiplexing techniques, it is important to ensure that the MZI path length imbalances are distinct to ensure that the frequency components do not overlap. Furthermore, the MZI path length imbalances must be chosen to ensure that the sum and difference frequency components present in the spectrum are not equal to the primary frequencies.

A change in local environmental parameters will result in a concomitant change in the effective refractive indexes of the core and cladding modes. This will be exhibited as a change in the phase of the interference fringes, which may be determined from analysis of the Fourier transform of the transmission spectrum $F(I)$. From the preceding plot, the various frequencies present in the spectrum are known. These frequencies were then isolated, and after translation to the origin, the arctangent of the ratio of the real to imaginary components was used to determine the phase. This process is outlined in [10]. The phase values obtained in this way were wrapped modulo $2 \pi$, resulting in discontinuities in the phase, which were then unwrapped using a simple algorithm.

\section{Experiment and Results}

To demonstrate the operation of the multiplexing scheme, two cascaded LPG pairs were fabricated in hydrogen-loaded single-mode optical fiber with a cutoff wavelength of $820 \mathrm{~nm}$ via UV irradiation though an amplitude mask with a period of $400 \mu \mathrm{m}$. For the first LPG pair, the buffer jacket was removed over a length of $130 \mathrm{~mm}$, and two 20-mm-long LPGs were fabricated with a physical separation of $90 \mathrm{~mm}$, producing the transmission spectrum shown in Fig. 4(a). The second LPG pair was fabricated $1 \mathrm{~m}$ further along the fiber, in a stripped section of fiber with a length of $60 \mathrm{~mm}$. The LPGs were $20 \mathrm{~mm}$ long, separated by $30 \mathrm{~mm}$. The transmission spectra were monitored by coupling the output from a tungsten-halogen lamp into the fiber and coupling the output of the fiber to a CCD spectrometer (Ocean Optics HR4000).

The influence of the separation of the LPGs on the frequency of the fringes is evident. The low visibility of the fringes in Fig. 4(a) may be a result of the attenuation of the cladding mode over the 90-mm-long fiber separating the LPGs. The transmission spectrum observed upon inscribing two such interferometers in a single optical fiber is shown in Fig. 5. This arrangement results in the envelope of the attenuation band being modulated by two distinct frequencies.

Thirty-millimeter-long sections of the length of fiber separating each LPG pair were immersed within cells containing Cargille oil with a refractive index of $\sim 1.46$. Each cell was mounted on a Peltier thermoelectric heater to independently control the temperature of the oil surrounding the fiber sections. As the temperature of the oil was varied, a concomitant change in the phase of the corresponding fringe pattern was observed, arising from the thermal expansion and thermooptic response of

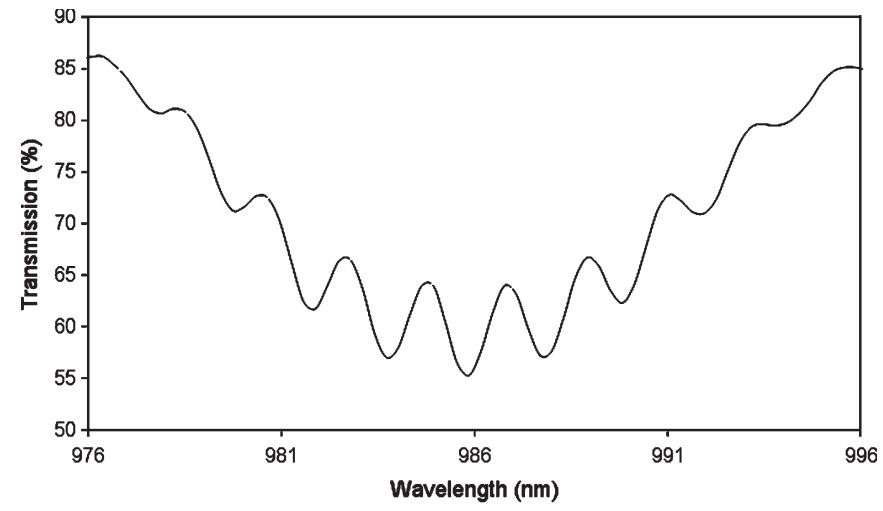

(a)

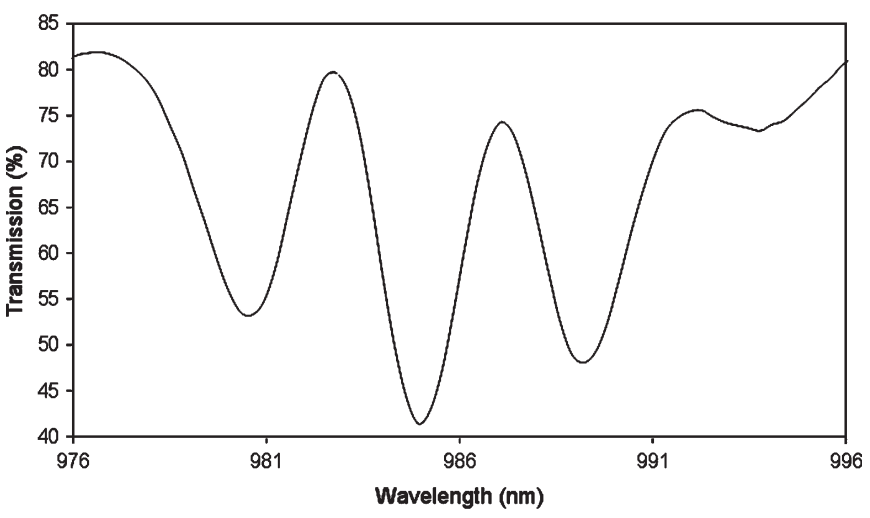

(b)

Fig. 4. (a) Transmission spectrum of a cascaded LPG pair with a separation of $90 \mathrm{~mm}$. (b) Transmission spectrum of a cascaded LPG pair with a separation of $30 \mathrm{~mm}$. The LPGs were $20 \mathrm{~mm}$ long, had a period of $400 \mu \mathrm{m}$, and were fabricated within a hydrogen-loaded single-mode optical fiber with a cutoff wavelength of $820 \mathrm{~nm}$.

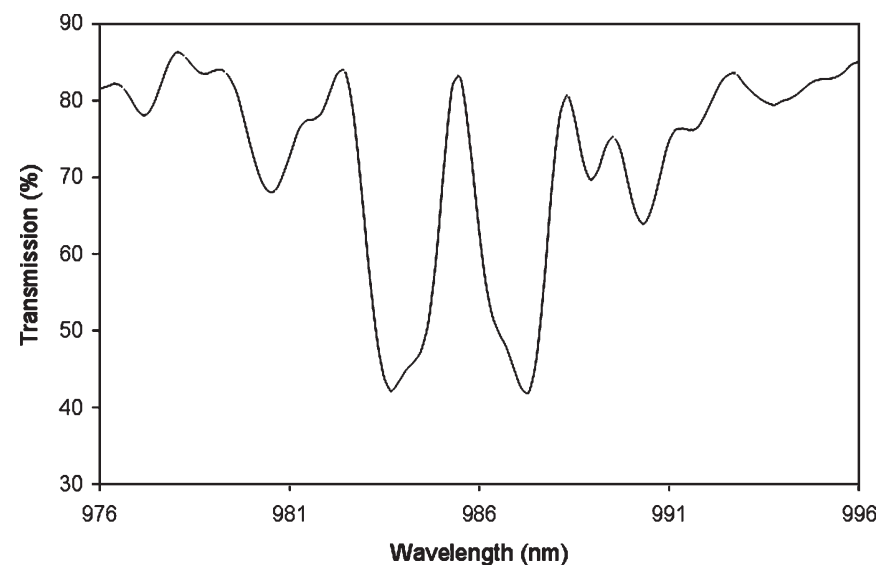

Fig. 5. Transmission spectrum of an optical fiber containing two cascaded LPG pairs, in which the LPGs were separated by 90 and $30 \mathrm{~mm}$, respectively.

the fiber and the change in effective index of the cladding mode caused by the thermally induced refractive index change of the oil. Below $\sim 40{ }^{\circ} \mathrm{C}$, the refractive index of the oil exceeded that of the cladding of the fiber, with the result that no fringes were observed in the transmission spectrum. As the temperature was increased beyond $40{ }^{\circ} \mathrm{C}$, the negative thermooptic coefficient of the oil caused the refractive index to decrease, allowing the cladding mode to propagate to the second LPG and in 


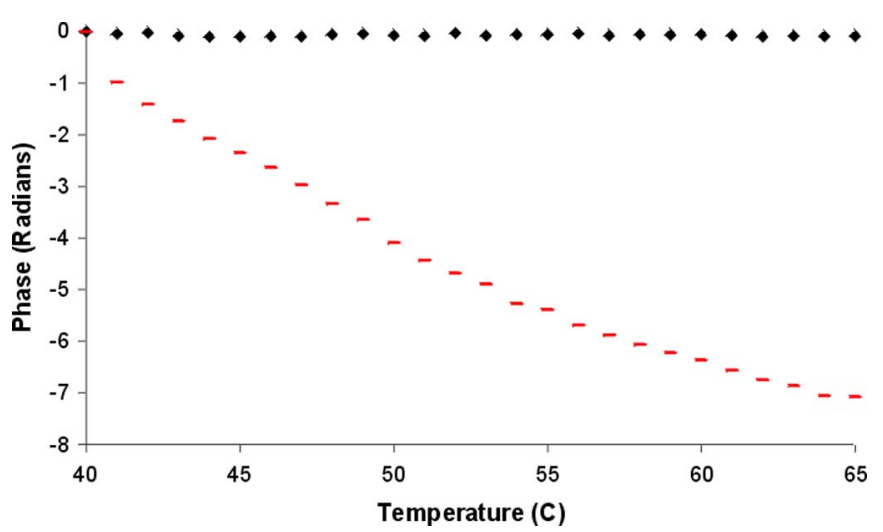

Fig. 6. Change of phase of the fringe patterns in response to increasing the temperature of Cargille oil surrounding a 40-mm-long section of the fiber separating the LPGs in LPG pair 1 (90-mm separation). fringes corresponding to LPG pair 1 (90-mm separation). -: phase of fringes corresponding to LPG pair 2 (30-mm separation).

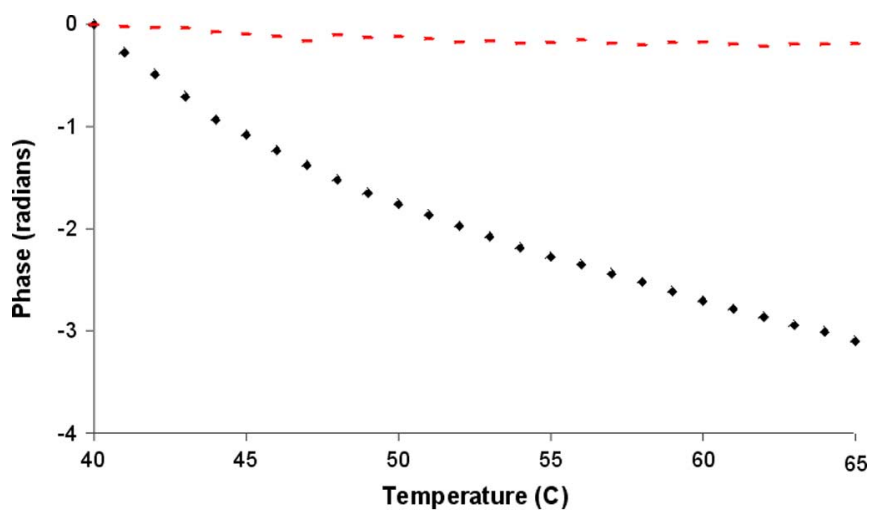

Fig. 7. Change of phase of the fringe patterns in response to increasing the temperature of Cargille oil surrounding a 20-mm-long section of the fiber separating the LPGs in LPG pair 2 (20-mm separation). fringes corresponding to LPG pair 1 (90-mm separation). -: phase of fringes corresponding to LPG pair 2 (20-mm separation).

turn producing the interference fringes. As the temperature was increased, the phase of the fringes responded to the change in temperature of the length of fiber submersed in the oil and to the change in refractive index of the oil, which modified the effective index of the cladding mode. The Fourier transform of the optical spectrum was analyzed to yield the phase change of each of the fringe patterns at a specified wavelength in the spectrum.

Figs. 6 and 7 show the change associated with the frequencies of the two fringe patterns, determined at a wavelength of $980 \mathrm{~nm}$, when the lengths of optical fiber immersed in the oil were heated. In Fig. 6, the temperature of the oil bath surrounding the fiber in LPG pair 1 (90-mm separation) was varied, while the oil bath for LPG pair 2 (30-mm separation) was held constant at $40{ }^{\circ} \mathrm{C}$. The fringes were observed to suffer a red-shift, and the phase analysis of the high-frequency fringes revealed an 8-rad variation over the temperature range. The drift in the phase of the lower frequency fringes, corresponding to LPG pair 2, equates to a variation of the temperature of the oil of $\pm 0.15{ }^{\circ} \mathrm{C}$ about a mean of $40{ }^{\circ} \mathrm{C}$, as determined from the data shown in Fig. 7. This lies within the temperature stability achieved by the thermoelectric controller used.

The experiment was repeated, heating the oil surrounding the fiber separating the LPGs in LPG pair 2 while holding the temperature of LPG pair 1 constant at $40{ }^{\circ} \mathrm{C}$. In this case, the lower frequency fringes corresponding to LPG pair 2 are observed to exhibit a significant phase change, while the phase of the fringes corresponding to LPG pair 1 show a small drift, which equates to a variation of $\pm 0.1^{\circ} \mathrm{C}$ around a mean of $40{ }^{\circ} \mathrm{C}$, as determined from the data shown in Fig. 6. This lies within the temperature stability achieved by the thermoelectric controller used.

\section{CONCLUSION}

The multiplexing of independent LPG-based MZIs has been demonstrated. The LPG pairs forming the MZIs were fabricated with different separations, producing fringe patterns of different frequencies. Fourier analysis of the transmission spectrum of the system allowed discrimination of the different frequencies and measurement of changes in the phase of the fringe patterns in response to external stimuli.

\section{REFERENCES}

[1] S. W. James and R. P. Tatam, "Optical fibre long period grating sensors: Characteristics and application," Meas. Sci. Technol., vol. 14, no. 5, pp. R49-R61, 2003.

[2] T. Allsop, T. Earthrowl, R. Reeves, D. J. Webb, and I. Bennion, "The interrogation and multiplexing of long period grating curvature sensors using a Bragg grating based, derivative spectroscopy technique," Meas. Sci. Technol., vol. 15, no. 1, pp. 44-48, 2004.

[3] B. H. Lee and J. Nishii, "Bending sensitivity of in-series long-period gratings," Opt. Lett., vol. 23, no. 20, pp. 1624-1626, Oct. 1998.

[4] O. Duhem, J. F. Henninot, and M. Douay, "Study of in fiber Mach-Zehnder interferometer based on two spaced 3-dB long period gratings surrounded by a refractive index higher than that of silica," Opt. Commun., vol. 180, no. 4-6, pp. 255-262, Jun. 2000.

[5] Y. G. Han, B. H. Lee, W. T. Han, U. C. Paek, and Y. Chung, "Fibre-optic sensing applications of a pair of long-period fibre gratings," Meas. Sci. Technol., vol. 12, no. 7, pp. 778-781, 2001.

[6] S. W. James, I. Ishaq, G. J. Ashwell, and R. P. Tatam, "Cascaded long period gratings with nano-structured coatings," Opt. Lett., vol. 30, no. 17, pp. 2197-2199, Sep. 2005.

[7] I. Sakai, "Frequency-division multiplexing of optical-fiber sensors using a frequency-modulated source," Opt. Quantum Electron., vol. 18, no. 4, pp. 279-289, 1986.

[8] I. Sakai, R. R. Youngquist, and G. Parry, "Multiplexing of optical fiber sensors using a frequency-modulated source and gated output," J. Lightw. Technol., vol. LT-5, no. 7, pp. 932-940, Jul. 1987.

[9] B. H. Lee, Y.-J. Kim, Y. Chung, W.-T. Han, and U.-C. Paek, "Fibre modal index measurements based on fibre gratings," Fiber Integr. Opt., vol. 20, no. 5, pp. 443-455, Sep. 2001.

[10] M. Takeda, H. Ina, and S. Kobayashi, "Fourier-transform method of fringe-pattern analysis for computer-based topography and interferometry," J. Opt. Soc. Amer., vol. 72, no. 1, pp. 156-159, Jan. 1982.

[11] S. Khaliq, S. W. James, and R. P. Tatam, "Enhanced sensitivity fibre optic long period grating temperature sensor," Meas. Sci. Technol., vol. 13, no. 5 , pp. 792-795, 2002.

Richard P. Murphy received the B.Sc. Joint Honours degree in mathematics/physics and the M.Sc. degree in mathematics from the National University of Ireland, Cork, Ireland, in 2002 and 2003, respectively.

He is currently a Research Student with the Engineering Photonics Group, Cranfield University, Cranfield, U.K. His current research interests include the development of sensors based on fiber-optic long-period gratings. 
Stephen W. James received the B.Sc. degree in physics and the M.Sc. degree in applied optics from Imperial College London, London, U.K., in 1987 and 1988, respectively, and the Ph.D. degree from University of Southampton, Southampton, U.K., in 1992.

$\mathrm{He}$ is currently a Senior Lecturer with the Engineering Photonics Group, Cranfield University, Cranfield, U.K. Since joining Cranfield University in 1993, he has developed interests in a range of optical measurement and sensing techniques, including laser anemometry, speckle interferometry, and optical fiber-grating-based sensors. He has published more than 150 papers in journals and conferences.

Dr. James is the Editor-in-Chief of Optics and Laser Technology.
Ralph P. Tatam received the B.Sc. degree in physics and chemistry and the D.Sc. degree from the University of Exeter, Exeter, U.K., in 1981, 1986, and 2005, respectively, and the Ph.D. degree in physics from the City of London Polytechnic, London, U.K., in 1986.

Following four years at the Physics Laboratory, University of Kent, he moved to Cranfield University, Cranfield, U.K., where he is currently a Professor of engineering photonics with the School of Engineering and heads the Engineering Photonics Group. He has published more than 300 papers and has been involved with numerous national and international conferences. He has been active in optical and optical-fiber-based instrumentation and sensing for 20 years. His current research interests include optical flow measurement, speckle interferometry, and optical fiber sensors.

Prof. Tatam is a Fellow of The International Society for Optical Engineering (SPIE). 